\title{
LinACE in-axis absolute linear encoder
}

\author{
Matjaž Sivec \\ RLS Merilna tehnika d.o.o., Pod vrbami 2, 1218 Komenda, Slovenia \\ matjaz.sivec@rls.si
}

\begin{abstract}
:
LinACE $^{\mathrm{TM}}$ is an extremely robust absolute linear cylindrical encoder system designed for integration into micro hydraulic, pneumatic and electromechanical actuators as a feedback element for position / velocity closed loop applications. The implementation of LinACE technology means progress from indirect position measurement done by a parallel encoder system to direct on-the-spot encoding by the novel in-axis absolute linear system.

The LinACE encoder consists of a sliding encoder readhead module and a solid steel encoder shaft which eliminates the need for a separate measurement system in motion applications, with numerous implications for design, complexity, cost-effectiveness and even feasibility of motion systems requiring accurate absolute position encoding.

The LinACE encoder is the result of an RLS d.o.o. project which has been partly funded by the European Union.
\end{abstract}

Key words: Absolute linear measurement, absolute linear encoder, absolute encoder, absolute position measurement, linear shaft encoder.

\section{Actuator system design}

Actuators are used in simple tasks where one actuator performs single movement as well as in highly sophisticated systems where multiple actuators perform well coordinated and controlled motion (e.g. industrial robots). Simple tasks are easily adjustable and usually require simple approaches to control the actuator position which is not the case in more complex integrated systems. Complex tasks usually have higher demands on the ability to control the position of the actuator and require a closed loop control.
An example of a high demanding application is laser welding which requires stability, accurate position and velocity control, or high performance medical applications where an electric actuator may be used as a piston pump to inject fluid into the patient or positioning of laser during eye operation.

Typical closed loop design comprises a linear encoder installed parallel to an actuator and linked to the piston shaft as shown in figure 1.

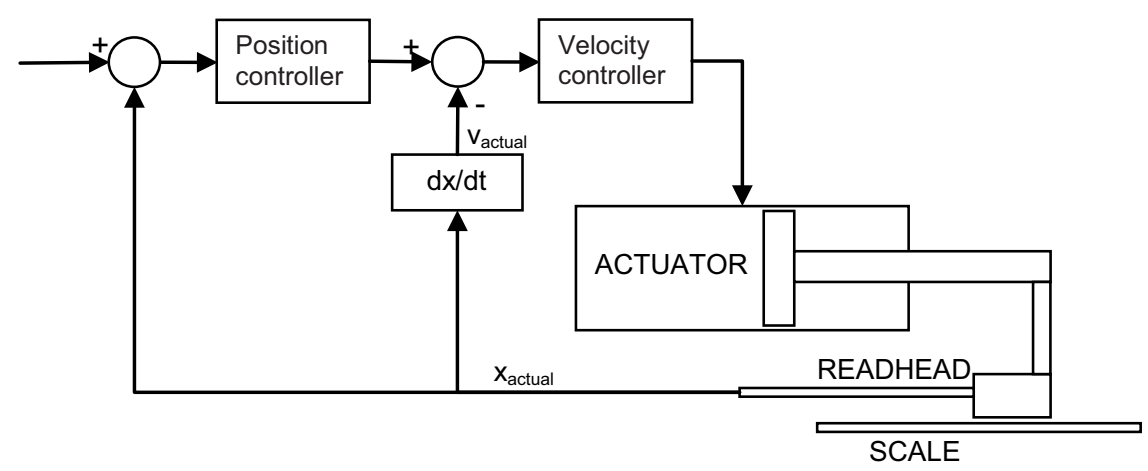

Fig. 1: Typical actuator system with linear scale position feedback control. 
In LinACE's case, the information carrier is a machine element (a piston shaft) of the actuator itself. This approach provides a number of advantages compared to existing absolute position measurement systems (miniaturisation, accuracy, reliability, robustness, reduction of system's components), and is based on a number of innovative patent pending technologies developed within this project.

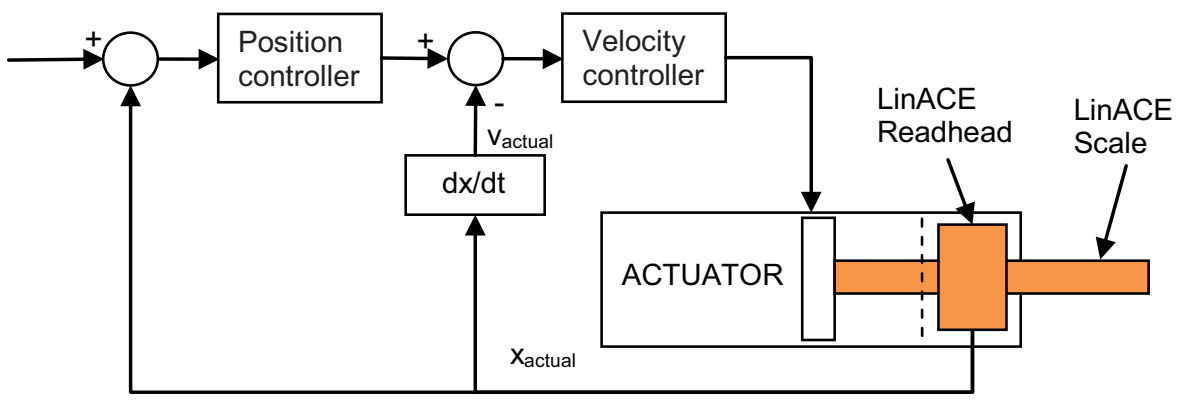

Fig. 2: Actuator system with LinACE position feedback control.

\section{How it works}

The LinACE technology is based on absolute code written into an information carrier in the form of regions with different magnetic permeability; which is then read by an array of Hall sensors integrated in a single silicon die.

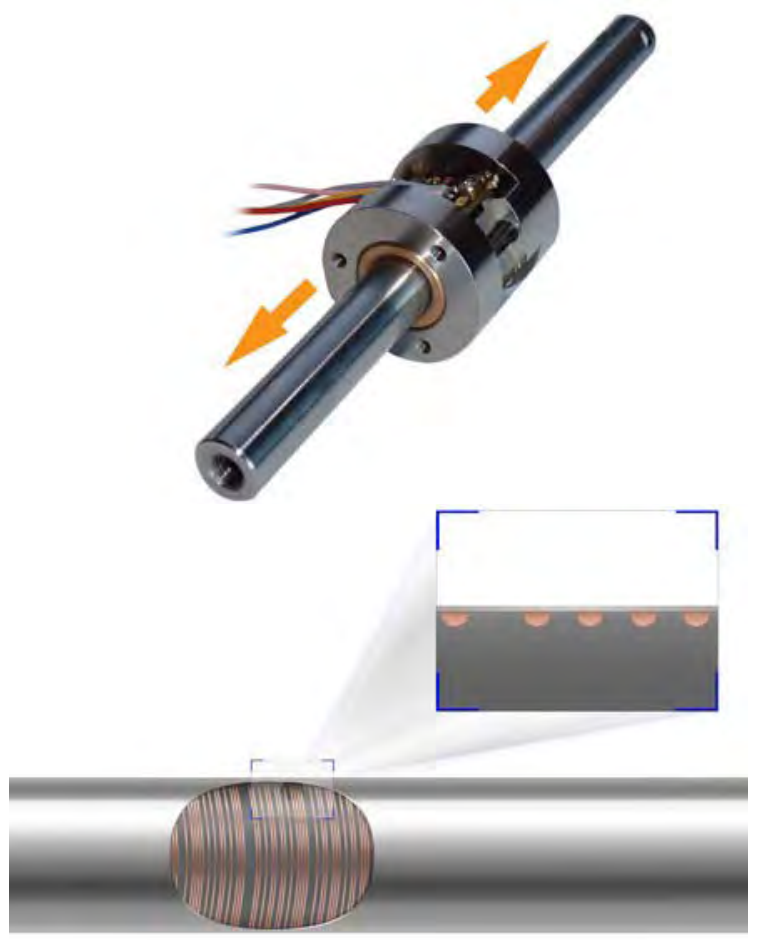

Fig. 3: The shaft is used as a load-bearing functional part of the motion system and as an information carrier read by the module with integrated bearings.
Grooves applied in the carbon solid steel shaft with high relative permeability represent pseudo-random binary sequence (PRBS absolute code). Grooves may be filled with hard chrome, copper or any other material with low permeability by galvanic or thermal spraying process.

Using a bias magnet, differences in magnetic permeability modulate magnetic field density, which is detected and converted to electrical signals by a Hall sensors array integrated in a single-silicon-die ASIC. Generated electrical signals are processed using algorithms including Fourier transform to calculate position based on millimeter-scale bit length down to sub-micrometer resolutions.
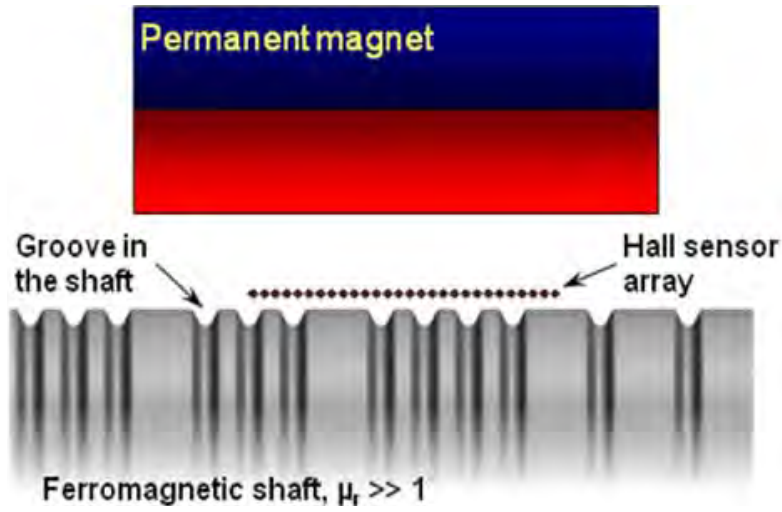

Fig. 4: Arrangement of permanent magnet, Hall sensor array and carbon steel shaft with groove sequence (above) and modulation of the magnetic field near the carbon steel shaft (next page). 


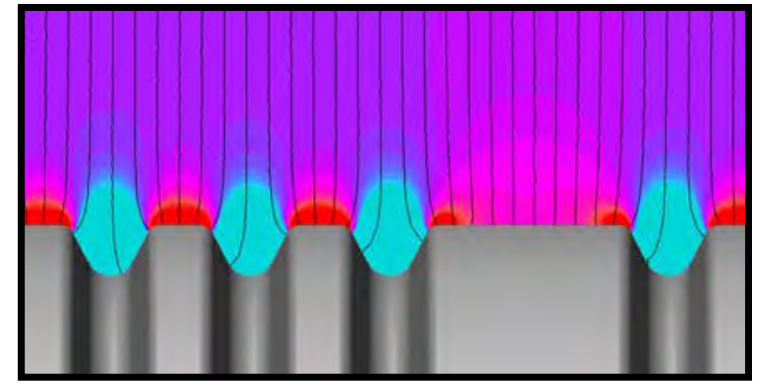

Magnetic field is detected only on a very limited distance $0.1 \pm 0.05 \mathrm{~mm}$. To achieve correct silicon die guiding on the coded shaft, module with integrated sliding bearings and processing electronics was developed.

Besides cutting the grooves and filling them by galvanic process there is also a more innovative way to create regions of material with low permeability. New procedures were developed to obtain a high content of martensitic structure in the substrate made of austenitic stainless steel (SST) material.

Martenistic structure has high permeability like carbon steel while austenitic material is nonmagnetic. If the SST with martensitic structure is heated above the austenitising temperature, the material structure is transformed back to austenitic. So the martensitic substrate is suitable for information writing by local thermal treatment. To achieve this transformation another innovative process of local thermal treatment by a laser beam has been developed by RLS d.o.o.

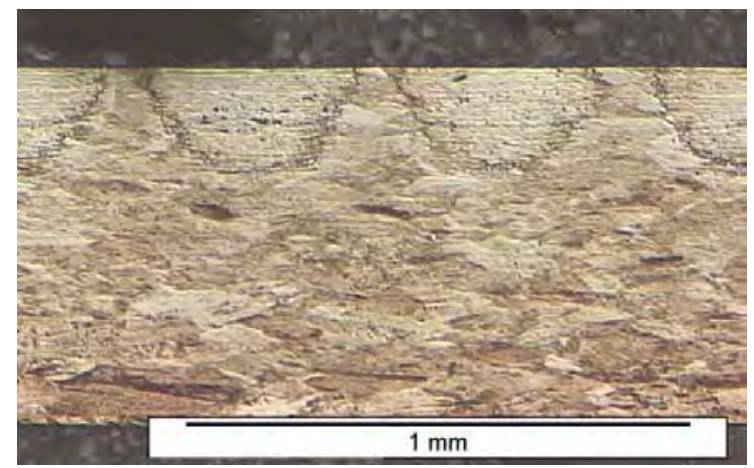

Fig. 5: Austenitic non magnetic regions made by laser local temperature treatment in the stainless steel shaft with high permeability martensitic structure.

\section{The advantages of the LinACE absolute linear encoder}

Going from parallel encoder systems to the inaxis absolute linear systems is a step from indirect measurement to direct on-the-spot encoding. On-the-spot encoding means greater accuracy and robustness as well as less space consumption and therefore enables to consider new possibilities of using linear actuators in industrial and medical systems.

LinACE encoder is a true absolute system for integration into an actuator which offers a range of selectable resolutions down to $0.5 \mu \mathrm{m}$ with velocities up to $5 \mathrm{~m} / \mathrm{s}$. Measuring length is available up to $500 \mathrm{~mm}$ for shaft diameter $4 \mathrm{~mm}$ to $16 \mathrm{~mm}$. The encoder readhead operates at temperatures from $-40{ }^{\circ} \mathrm{C}$ to $+85{ }^{\circ} \mathrm{C}$ and is resistant to shock and vibrations. The system is unaffected by dirt, dust, liquids and stray magnetic fields, and is also corrosion resistant.

LinACE offers a wide compatibility to existing controllers on the market due to a variety of output options (CAN, SSI, PWM and Asynchronous serial). 